\author{
Military Technical College \\ Kobry El-Kobbah, \\ Cairo, Egypt.
}

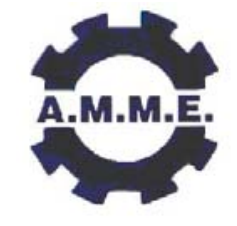

$13^{\text {th }}$ International Conference on Applied Mechanics and Mechanical Engineering.

\title{
EVALUATION OF FRICTIONAL CHARACTERISTICS OF AEROSTATIC LINER BEARING
}

\author{
FUJII $Y$.
}

\begin{abstract}
A method based on the Levitation Mass Method (LMM) for evaluating the components of the force acting on a moving part of a pneumatic linear bearing has been proposed by the author. The total force acting on the moving part is accurately measured as the inertial force using an optical interferometer. The efficiency of the developing method for evaluating the frictional characteristics of pneumatic linear bearings and the new information obtained on the frictional characteristics of a pneumatic linear bearing is discussed. The essence of the LMM as a precision method for measuring mechanical quantities is reviewed.
\end{abstract}

\section{KEY WORDS}

inertial force, inertial mass, optical interferometer, pneumatic linear bearing, friction, tribology.

\footnotetext{
* Professor, Dpt. of Electronic Engineering, Gunma University, Kiryu, Japan.
} 


\section{INTRODUCTION}

Aerostatic linear bearings have the two major features that are high accuracy in motion and low friction. For the former feature, i.e. high accuracy in motion, these bearings and linear motors are widely used in combination in the most precise machine tools and instruments in which precise linear motion is required. There have been some studies on this feature. The latter feature, i.e. low friction, has not attracted the close attention of engineers and researchers in the field. However it will attract the interest for improving the accuracy of motion control in the near future.

A method [1-3] for evaluating forces acting on the moving parts of pneumatic linear bearings is reviewed. In the method, only the Doppler sift frequency of the laser beam reflected on the moving part of a pneumatic linear bearing is measured using the optical interferometer. The velocity, the position, the acceleration, and the inertial force are calculated from the frequency. Then the components of the force, such as the force component depending on position, the force component depending on velocity, and the force component depending on tilt angle of the guide-way, are evaluated using the least squares method. Using the developing method, the frictional characteristics of a pneumatic linear bearing were evaluated in detail.

In the previously reported experiment [2,3], the component of dynamic frictional force, which is almost independent of the absolute value of velocity, was detected in the experiment, in addition to the force component that is proportional to velocity. Before that study [2,3], dynamic frictional force had only been explained as the viscous frictional force acting inside the air film, which is proportional to the velocity.

The developing method can be also applied to evaluate the frictional characteristics of linear ball bearings [4]. In addition, the developing method can be applied to evaluate the frictional characteristics of other materials [5-7].

The proposed method is based on the Levitation Mass Method, which has been proposed and developed by the author. In the LMM, the inertial force of a mass levitated using a pneumatic linear bearing is used as the reference force applied to the objects under test, such as force transducers, materials or structures. The inertial force of the levitated mass is measured using an optical interferometer. The author has modified it as calibration methods for all the three categories of the dynamic force calibration [8-10], that are the dynamic calibration method under impact load [8], the dynamic calibration method under oscillation load [9] and the dynamic calibration method under step load [10]. The authors have applied the LMM for material testing, such as methods for evaluating material viscoelasticity under an oscillating load [11] and under an impact load [12,13], a method for evaluating biomechanics [14-17], a method for evaluating dynamic response of impact hammers [18] and a method for generating and measuring a micro-Newton level forces [19-22]. To improve the efficiency of the LMM, a pendulum mechanism [23] for use as a substitute of a pneumatic linear bearing and a frequency measurement technique [24,25] using a digitizer instead of an electronic frequency counter have been developed. Recently, a method for correcting the effect of the inertial mass on dynamic force measurements has been proposed based on the LMM $[26,27]$. 
The efficiency of the developing method for evaluating the frictional characteristics of pneumatic linear bearings and the new information obtained on the frictional characteristics of a pneumatic linear bearing is discussed in this paper.

\section{EXPERIMANTAL SETUP}

Figure 1 shows the experimental setup for measuring the force acting on the moving part of an aerostatic linear bearing. A linear bearing is mounted on a tilting stage whose tilt angle is adjustable. In the experiment, the initial velocity of the moving part is given to the moving part by hand. Then the moving part begins to move back and forth between the two dampers. The velocity of the moving part is accurately measured using an optical interferometer and electric frequency counters.

The total force acting on the moving part is measured as the product of mass and acceleration, $\boldsymbol{a}(\mathrm{m} / \mathrm{s} 2)$. The acceleration is calculated by differentiating the velocity, $\boldsymbol{v}$ $(\mathrm{m} / \mathrm{s})$, with respect to time. The velocity is measured as the Doppler shift frequency of the signal beam of a laser interferometer, fDoppler, which can be expressed as

$\boldsymbol{v}=\lambda_{\text {air }}\left(f_{\text {Doppler }}\right) / 2$

$f_{\text {Doppler }}=-\left(f_{\text {beat }}-f_{\text {rest }}\right)$,

where $\lambda_{\text {air }}$ is the wavelength of the signal beam under the experimental conditions, $\boldsymbol{f}_{\text {beat }}$ is the beat frequency which is the frequency difference between the signal beam and the reference beam, $\boldsymbol{f}_{\text {rest }}$ is the rest frequency which is the value of $\boldsymbol{f}_{\text {beat }}$ when the moving part is at standstill.

A Zeeman-type two-frequency He-Ne laser is used as the light source. The frequency difference between the signal beam and the reference beam, i.e., the beat frequency, $\boldsymbol{f}_{\text {beat, }}$, is measured from an interference fringe which appears at the output port of the interferometer; it varied around $\boldsymbol{f}_{\text {rest }}$, approximately $2.9 \mathrm{MHz}$, depending on the velocity of movement. An electric frequency counter (model: R5363; manufactured by Advantest Corp., Japan) continuously measures and memorized the beat frequency, $\boldsymbol{f}_{\text {beat }}(\mathrm{Hz}), 1000$ times with a sampling interval of $\boldsymbol{T}=40000 / \boldsymbol{f}_{\text {beat }}(\mathrm{s})$. In other words, it continuously measures the time of every 40000 periods without dead time, calculates the frequency and stores the 1000 values in memory. The sampling period of the counter is approximately $14 \mathrm{~ms}$ at the frequency of $2.9 \mathrm{MHz}$. Another electric counter (model TA1100, manufactured by Yokogawa Corp., Japan) is measured the rest frequency, $\boldsymbol{f}_{\text {resr }}(\mathrm{Hz})$, using an electric signal supplied by a photo diode embedded inside the He-Ne laser.

Measurement of the electric counter (R5363) is triggered by means of a light switch, i.e. a combination of a laser-diode and photo-diode. The total mass of the moving part, including the cube corner prism, is approximately $21.17 \mathrm{~g}$.

The aerostatic linear bearing, "GLS08A50/25-2571" (NSK Co., Ltd., Japan), is attached to an adjustable tilting stage. The stroke of the movement is approximately $25 \mathrm{~mm}$ and the nominal thickness of the air film is approximately $10 \mathrm{~mm}$. 


\section{RESULT}

Figure 2 shows data processing procedure of calculating the velocity, the position, the acceleration and the force from the frequency. In the experiment, only the beat frequency, $\boldsymbol{f}_{\text {beat }}$, and the rest frequency, $\boldsymbol{f}_{\text {rest }}$, are measured using the optical interferometer. The velocity, $\boldsymbol{v}$, the position $\boldsymbol{x}$, the acceleration, $\boldsymbol{a}$, and the force, $\boldsymbol{F}$, are calculated from the beat frequency, $\boldsymbol{f}_{\text {beat }}$, and the rest frequency, $\boldsymbol{f}_{\text {rest. }}$ In the experiment, the moving part collides with the left-side damper two times and with the right-side damper one time before it stops between the dampers due to the friction acting inside the bearing.

Figure 3 shows the magnified figure, which shows the change in force $\boldsymbol{F}$ against time $\boldsymbol{t}$, shown in Figure 2. As seen form the figure, the small force at the micro-Newton level, which is the total force acting on the moving part, is clearly measured.

Figure 4 shows the change in force against position. The dependency of force on position is clearly observed. This is though to be due to the asymmetry of the airflow in the air film between the moving part and the guide part.

Figure 5 shows the change in force against velocity. The dependency of force on velocity is clearly observed. This is though to be due to the dynamic friction acting inside the air film between the moving part and the guide part.

\section{DISCUSSIONS}

Using this method, the force acting on the moving part of a aerostatic linear bearing can be accurately measured. This method will be effective to improve the knowledge on the dynamics of the aerostatic linear bearing. It will be useful for inspection of the manufacturing process of air bearings.

The advantages of the LMM as a precision mechanical measurement method are discussed with developed applications and possible applications. The author believes that the LMM is one of the keys to improve the field of the precision mechanical measurement, including the tribology of aerostatic linear bearings.

\section{CONCLUSIONS}

The essence of the Levitation Mass Method (LMM) as a precision method for measuring mechanical quantities is reviewed. The efficiency of the developing method for evaluating the frictional characteristics of pneumatic linear bearings is shown. The new information obtained on the frictional characteristics of a pneumatic linear bearing is reported.

\section{ACKNOWLEDGEMENT}

This work was supported by Grant-in-Aid for Scientific Research (B) 19360185 (KAKENHI 19360185). 


\section{REFERENCES}

[1] Y. Fujii and H. Fujimoto, "Measurements of frictional characteristics of a pneumatic linear bearing", Meas. Sci. Technol., Vol.10, No.5, pp.362-366, 1999.

[2] Y. Fujii, "Measurement of force acting on a moving part of a pneumatic linear bearing", Rev. Sci. Instrum., Vol.74, No.6, pp.3137-3141, 2003.

[3] Y. Fujii, "Frictional characteristics of an aerostatic linear bearing", Tribol. Int., Vol.39, No.9, pp.888-896, 2006.

[4] Y. Fujii, "An optical method for evaluating frictional characteristics of linear bearings", Optics and Lasers in Engineering, Vol.42, No.5, pp.493-501, 2004.

[5] Y. Fujii and T. Yamaguchi, "Optical method for evaluating material friction", Meas. Sci. Technol., Vol. 15, No.10, pp. 1971-1976, 2004.

[6] Y. Fujii and T. Yamaguchi, "Dynamic characteristics measurements of a car wiper blade", JSME Int. J. C, Vol.49, No.3, pp.799-803, 2006.

[7] Y. Fujii, "Method for measuring transient friction coefficients for rubber wiper blades on glass surface", Tribol. Int., Vol.41, No.1, pp.17-23, 2008.

[8] Y. Fujii, "Measurement of steep impulse response of a force transducer", Meas. Sci. Technol., Vol. 14, No.1 pp. 65-69, 2003.

[9] Y. Fujii, "A method for calibrating force transducers against oscillation force", Meas. Sci. Technol., Vol. 14, No.8, pp. 1259-1264,2003.

[10] Y. Fujii, "Proposal for a step response evaluation method for force transducers", Meas. Sci. Technol., Vol. 14, No.10, pp. 1741-1746, 2003.

[11] Y. Fujii and T. Yamaguchi, "Method for evaluating material viscoelasticity", Rev. Sci. Instrum., Vol.75, No.1, pp.119-123, 2004.

[12] Y. Fujii and T. Yamaguchi, "Proposal for material viscoelasticity evaluation method under impact load ", Journal of Materials Science, Vol.40, No.18, pp.4785 - 4790, 2005.

[13] Y. Fujii and D.W. Shu, "Impact force measurement of an actuator arm of a hard disk drive", Int. J. Impact Eng., Vol.35, No.2, pp.980108, 2008.

[14] Y. Fujii, T. Yamaguchi and J. Valera, "Impact response measurement of a human arm", Experimental Techniques, Vol.30, No.3, pp.64-68, 2006.

[15] Y. Fujii and T. Yamaguchi, "Method of evaluating the force controllability of human finger", IEEE Trans. Instrum. Meas., Vol.55, No.4, pp.1235-1241, 2006.

[16] Y. Fujii and K. Shimada, "Instrument for measuring the mass of an astronaut", Meas. Sci. Technol., Vol.17, No.10, pp.2705-2710, 2006.

[17] Y. Fujii and K. Shimada, "The space scale: An Instrument for astronaut mass measurement", Trans. Jpn. Soc. Aeronaut. Space Sci., Vol.50, No.170, pp.251-257, 2008.

[18] Y. Fujii, "Optical method for accurate force measurement: dynamic response evaluation of an impact hammer", Optical Engineering, Vol. 45, No. 2, 0230021-7, 2006.

[19] Y. Fujii, "Method for generating and measuring the micro-Newton level forces", Mech. Syst. Signal Pr., Vol.20, No.6, pp.1362-1371, 2006.

[20] Y. Fujii, "Microforce materials tester", Rev. Sci. Instrum., Vol.76, No.6, 0651111-7, 2005.

[21] Y. Fujii, "Microforce materials tester based on the levitation mass method", Meas. Sci. Technol., Vol.18, No.6, pp.1678-1682, 2007.

[22] Y. Fujii, "Method of generating and measuring static small force using downslope component of gravity", Rev. Sci. Instrum., Vol.78, No.6, 066104-1-3, 2007. 
[23] Y. Fujii, "Pendulum for precision force measurement”, Rev. Sci. Instrum., Vol. 77, No.3, 035111-1-5, 2006.

[24] Y. Fujii and J. Valera, "Impact force measurement using an inertial mass and a digitizer", Meas. Sci. Technol., Vol.17, No.4, pp. 863-868, 2006.

[25] Y. Fujii, "Impact response measurement of an accelerometer", Mech. Syst. Signal Pr., Vol.21, No.5, pp.2072-2079, 2007.

[26] Y. Fujii, "Measurement of the electrical and mechanical responses of a force transducer against impact forces", Rev. Sci. Instrum., Vol.77, No.8, 085108-1-5, 2006.

[27] Y. Fujii, "Method for correcting the effect of the inertial mass on dynamic force measurements", Meas. Sci. Technol., Vol.18, No.5, pp.N13-N20, 2007.

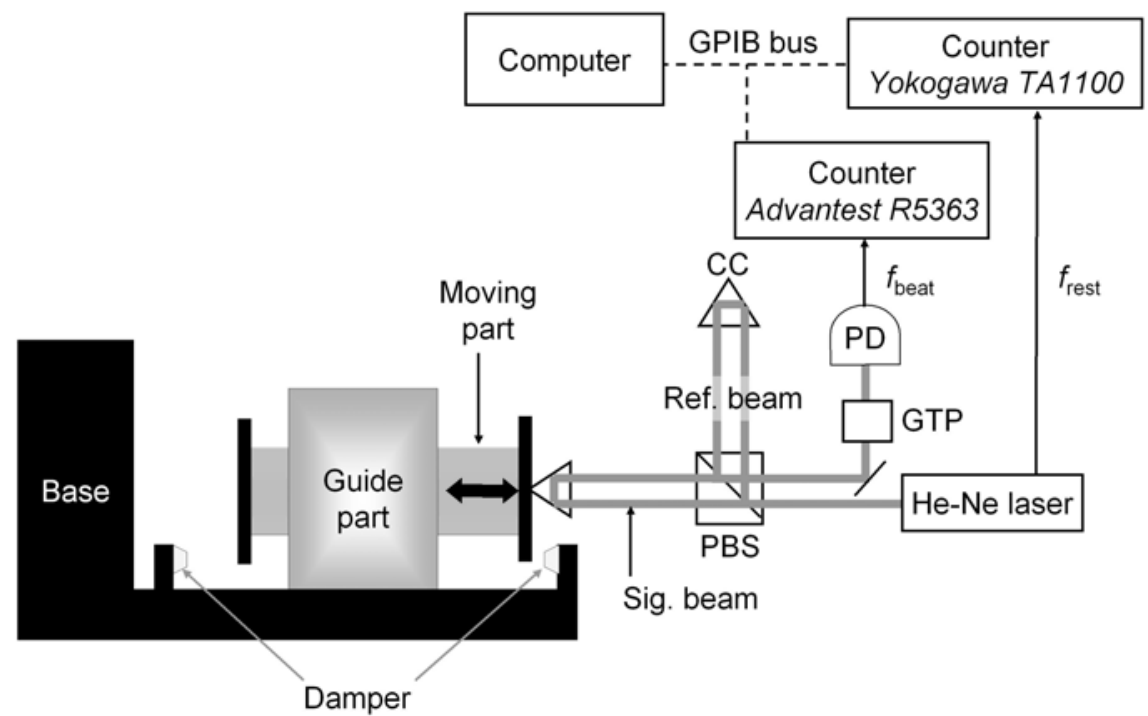

Figure 1 Experimental Setup
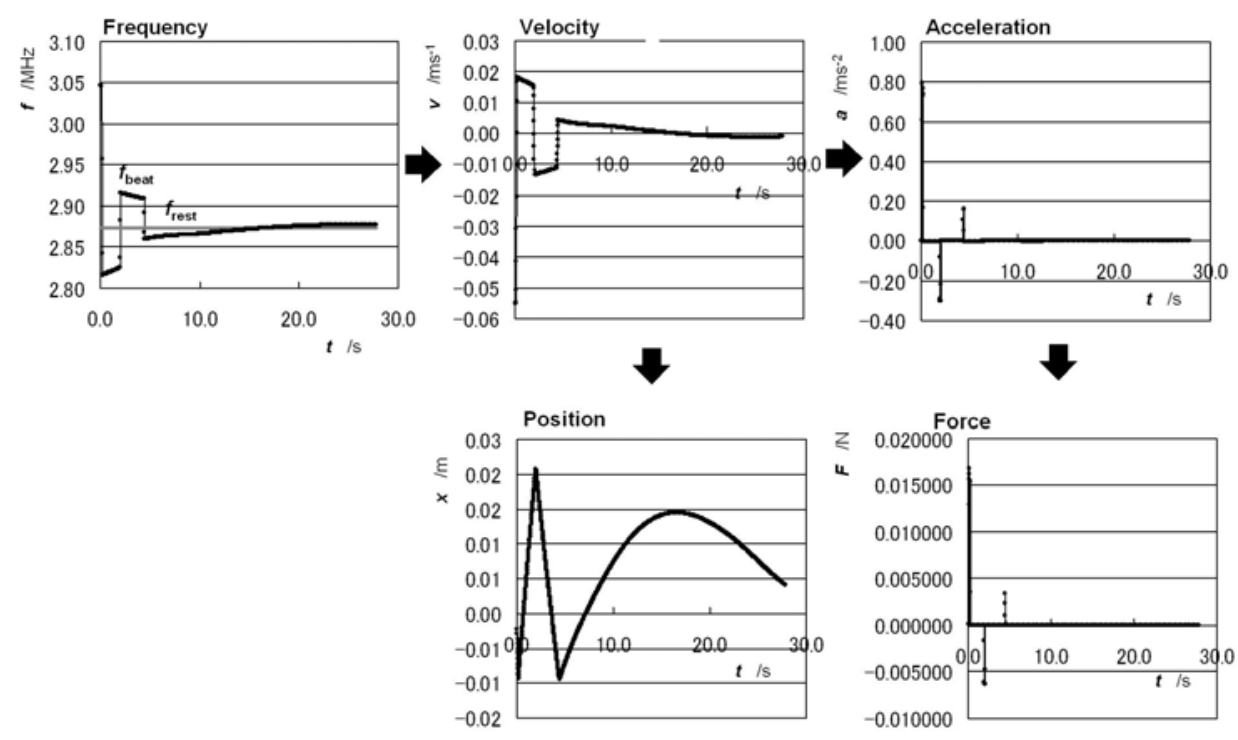

Figure 2. Data processing procedure: 


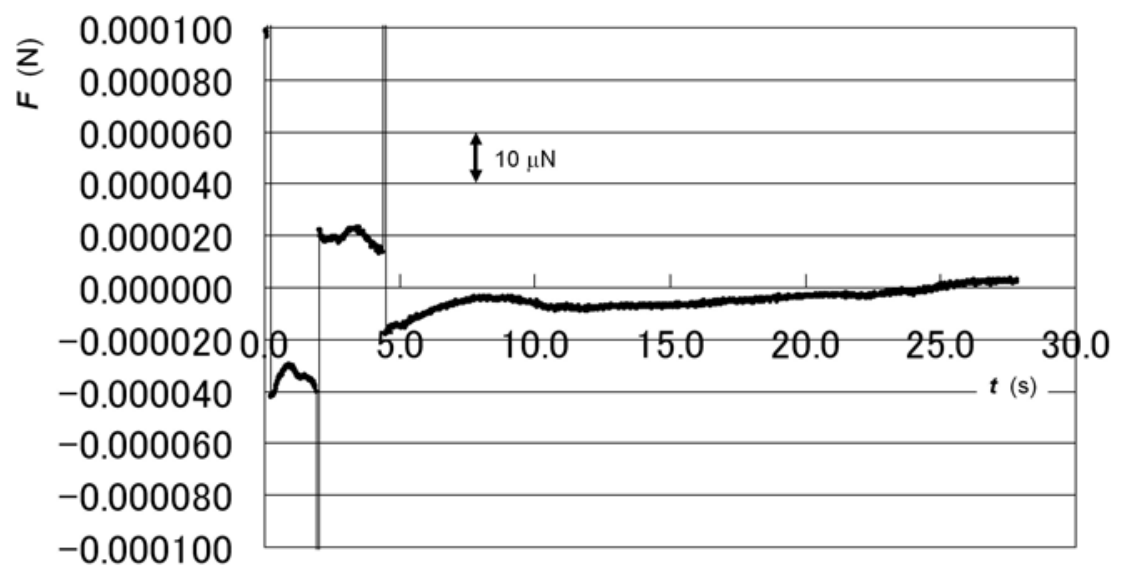

Figure 3. Change in force $\boldsymbol{F}$ against time $\boldsymbol{t}$

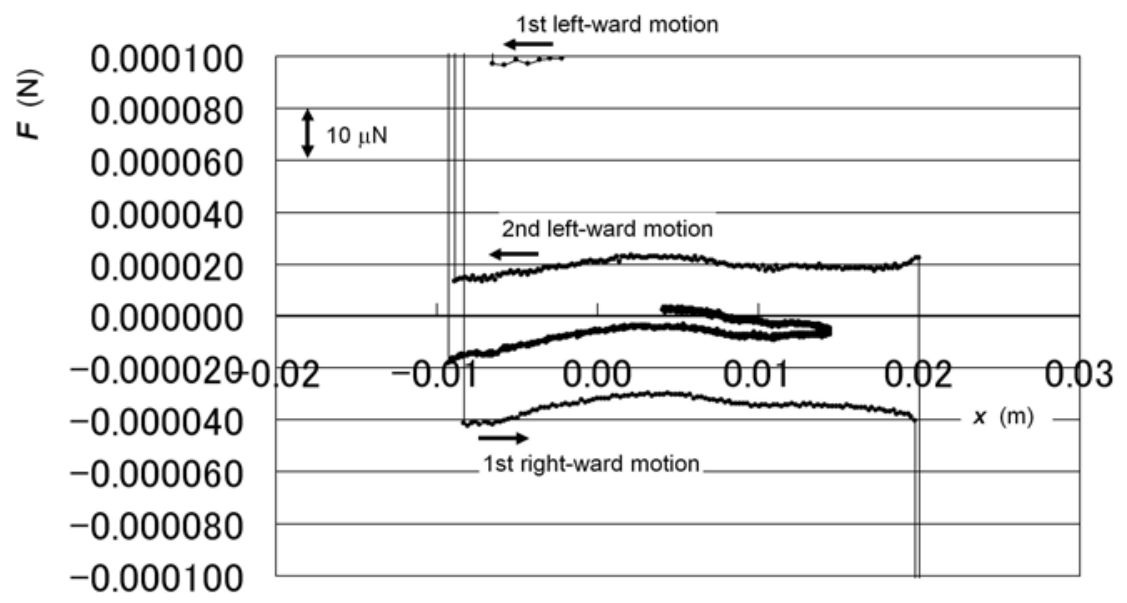

Figure 4. Change in force $F$ against position $x$

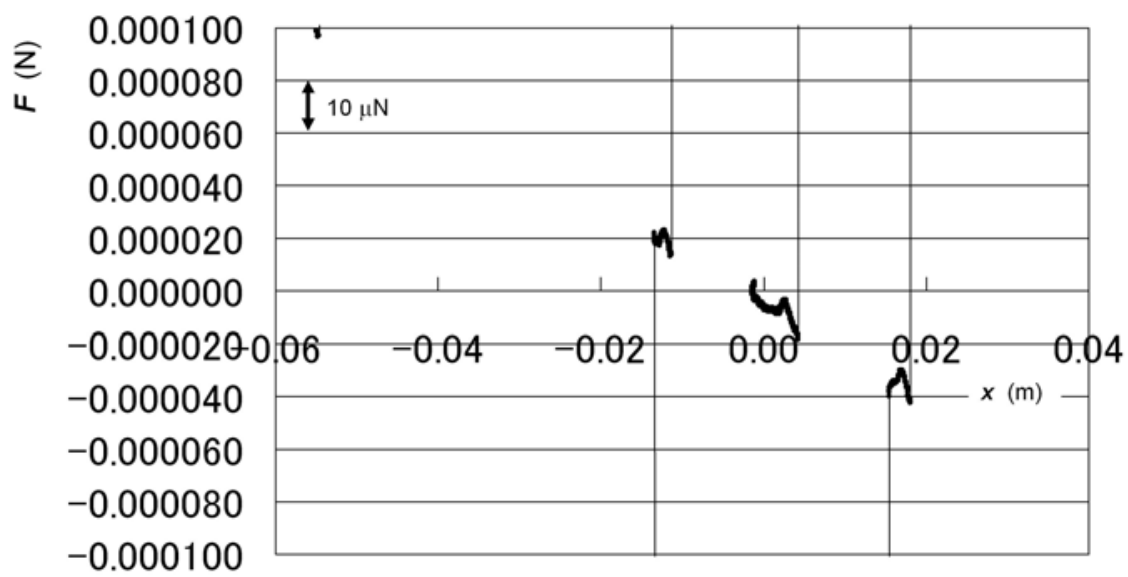

Figure 5. Change in force $\boldsymbol{F}$ against velocity $\boldsymbol{v}$ 